MITSUBISHI ELECTRIC RESEARCH LABORATORIES

http://www.merl.com

\title{
Distributed Three-phase Reactive Power Control of Distributed Energy Resources in Distribution Systems
}

\author{
Sun, H.; Benosman, M.; Nikovski, D.; Zhang, J.; Takano, T.; Kojima, Y.; Ohno, T.
}

TR2014-098 October 2014

\begin{abstract}
This paper proposes a new distributed control method for three-phase reactive power control of distributed energy resources (DERs) in distribution systems. Each DER-connected bus has been equipped with a local bus controller which has bi-way communications with bus controllers at adjacent buses upstream and downstream to the bus under consideration. The reactive powers of a DER-connected bus are adjusted to prevent its monitored buses from voltage violations. When voltage violations occur, the required reactive powers for the bus are determined based on the voltage violations on its monitored buses, and equivalent impedances and phase angle shifting between the substation transformer and the study bus. If the required reactive powers of a DER-connected bus are beyond its capacities, the neighbor DER-connected buses are required to share the additional generations. A new iterative residual splitting distributed control algorithm is proposed to coordinate the reactive powers between DER-connected buses. Each DER unit at a DER-connected bus has been equipped with a local unit controller which has bi-way communications with unit controllers at adjacent units of the same bus as the unit under consideration. A consensus-based distributed control algorithm is used to allocate the total demand among units with respect to their capacities. The test results on a modified IEEE 123-node feeder are given to demonstrate the effectiveness of the proposed method.
\end{abstract}

IEEE PowerCon 2014

This work may not be copied or reproduced in whole or in part for any commercial purpose. Permission to copy in whole or in part without payment of fee is granted for nonprofit educational and research purposes provided that all such whole or partial copies include the following: a notice that such copying is by permission of Mitsubishi Electric Research Laboratories, Inc.; an acknowledgment of the authors and individual contributions to the work; and all applicable portions of the copyright notice. Copying, reproduction, or republishing for any other purpose shall require a license with payment of fee to Mitsubishi Electric Research Laboratories, Inc. All rights reserved.

Copyright (C) Mitsubishi Electric Research Laboratories, Inc., 2014

201 Broadway, Cambridge, Massachusetts 02139 



\title{
Distributed Three-phase Reactive Power Control of Distributed Energy Resources in Distribution Systems
}

\author{
Hongbo Sun, Senior Member, IEEE, Mouhacine Benosman, Senior Member, IEEE, Daniel Nikovski, \\ Member, IEEE, Jinyu Zhang, Fellow, IEEE, Tomihiro Takano, Yasuhiro Kojima, Member, IEEE and \\ Tetsufumi Ohno
}

\begin{abstract}
This paper proposes a new distributed control method for three-phase reactive power control of distributed energy resources (DERs) in distribution systems. Each DER-connected bus has been equipped with a local bus controller which has bi-way communications with bus controllers at adjacent buses upstream and downstream to the bus under consideration. The reactive powers of a DER-connected bus are adjusted to prevent its monitored buses from voltage violations. When voltage violations occur, the required reactive powers for the bus are determined based on the voltage violations on its monitored buses, and equivalent impedances and phase angle shifting between the substation transformer and the study bus. If the required reactive powers of a DER-connected bus are beyond its capacities, the neighbor DER-connected buses are required to share the additional generations. A new iterative residual splitting distributed control algorithm is proposed to coordinate the reactive powers between DER-connected buses. Each DER unit at a DER-connected bus has been equipped with a local unit controller which has bi-way communications with unit controllers at adjacent units of the same bus as the unit under consideration. A consensus-based distributed control algorithm is used to allocate the total demand among units with respect to their capacities. The test results on a modified IEEE 123-node feeder are given to demonstrate the effectiveness of the proposed method.
\end{abstract}

Index Terms - Distributed control; distributed energy resources; distribution systems; reactive power control; three-phase

\section{INTRODUCTION}

$\mathrm{D}$ ISTRIBUTED energy resources (DER) is defined as smaller-scale power generation or storage, such as solar photovoltaic, wind farms, and plug-in hybrid electric vehicles[1]. The high penetration of DERs in distribution

Manuscript received July 10, 2014.

Hongbo Sun, Mouhacine Benosman, Daniel Nikovski, and Jinyun Zhang are with the Mitsubishi Electric Research Laboratories, Cambridge, MA 02139 USA.

Yasuhiro Kojima, Tomihiro Takano, and Tetsufumi Ohno are with the Mitsubishi Electric Corporation, Hyogo 661-8661 Japan. systems may cause system-wide voltage rise, or drop problems. Voltage violations are traditionally handled by automatically controlled tap-changing under load transformers, set voltage regulators, and fixed/switched capacitors. However, existing equipment is not inherently designed to handle the variability introduced by DERs, and the lifetime of the equipment could be dramatically reduced due to the increased number of operations that they may undergo.

A potential solution to this problem lies on the utilization of DERs to provide reactive power support for voltage regulation with proper control strategies. The controlling of system voltages through DERs can be achieved through either a centralized method [2]-[4], or a decentralized method [5]-[7]. The first method uses a centralized controller located at a substation to regulate all DERs in the system based on system-wide voltage measurements and complete information of DERs in the system. Although the centralized control methods can effectively regulate the system voltages, those methods required a complicated communication network for real-time applications, and any fault or delay in communication at a bus or DER can affect the performance of overall voltage regulation. The second method, which should be more economical and does not require complete knowledge of DERs, relies on a decentralized control strategy. Each distributed resource exchanges information with its neighbors and makes a local control decision. Collectively, local control decisions should have the same effect as the centralized control strategy. The authors of [5] proposed a switching control scheme where the DERs are operated with a constant power factor while bus voltages are within specifications, and, whenever there is a voltage violation, their reactive power is adjusted so that the system returns to the desired operating conditions. In [6] a multi-agent based decentralized approach was proposed for reactive power control of distributed resources, and the proposed approach was focused on linear consensus algorithms that converge to a cooperative solution in finite time. The authors of [7] proposed a two-stage voltage control architecture 
that the local controller for each bus first tries to adjust its reactive powers within its capacity to maintain its voltage within specifications whenever there is a voltage violation, and if additional capacities required, neighboring buses are required to support the additional request through a consensus based distributed algorithm. However, the sensitivity factors used for determining reactive power changes are based on Jacobian matrix which requires real-time measurements or estimations for system-wide voltage information, and those sensitivities are also sensitive to operating condition changes.

This paper proposes a new distributed control method for three-phase reactive power control of distributed energy resources in distribution systems. Each DER-connected bus is equipped with a bus controller to adjust the total reactive-power generations of distributed resources units to prevent all its monitored buses from voltage violations. When voltage violations occur, the required reactive power changes for a DER-connected bus are determined based on the voltage violations on its monitored buses, and equivalent impedances and phase angle shifting between the equivalent source at the substation and the study bus. If the required generations of a DER-connected bus are beyond its capacities, the neighbor DER-connected buses are joining in to share the required additional generations. The bus controller only needs communicate with its neighbor bus controllers located at its upstream and downstream, and uses locally measured information. Each DER unit at a DER-connected bus is equipped with a unit controller, and uses only local information and communicates with its neighbor units to determine its share of total reactive power changes required by the connected bus. A new iterative residual splitting algorithm is proposed to determine the reactive power changes of buses, and a consensus based algorithm is used to determine the share of each unit for the total reactive power changes at the bus.

\section{Determination of Three Phase Reactive Powers For DER-CONNECTED BUSES OF DISTRIBUTION SYSTEMS}

Fig. 1 shows an example of a distribution system. The distribution system includes a distribution substation in which a three-phase transformer receives electric power from power transmission systems, and provides the power to downstream loads through power lines. The distribution system may have several buses connected with distributed energy resources (DERs). The loads, DERs and power lines may be of single-phase, double-phase, or three-phase. The distribution systems operate in a radial configuration.

In Fig. 1, the system includes one substation transformer, and six DER-connected buses, including buses $D G_{1}, D G_{2}, D G_{3}$, $D G_{4}, D G_{5}$, and $D G_{6}$. Each bus may be connected with several distributed energy units. Taken bus $D G_{1}$ as example, it has connected with four photovoltaic units.

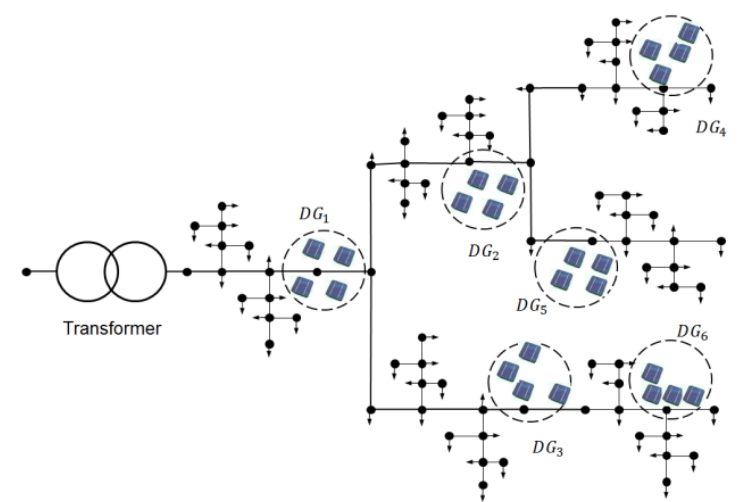

Fig. 1. An example of distribution system with DERs

Considering the characteristics of DERs, the DERs are well suitable for dealing with fast voltage variations caused by intermittent renewable resources and storage-capable loads, and not best fit for the slow voltage variation caused by regular load and generation changes which usually be handled by the substation transformers, voltage regulators and shunt capacitors. Therefore, it is assumed that the tap positions of transformers, regulators and energized capacitor units are fixed while regulating of distributed energy resources.

The reactive power generations of each DER-connected bus are regulated according to the measured voltages at a set of buses that monitored by the DER-connected bus. If the voltages on any phase of its monitored buses are sensed to be above or below the thresholds, an estimate for reactive power that should be injected into the bus to clear the voltage violation will be determined, and the corresponding reactive power changes for the DER-connected bus are obtained by averaging of required reactive power changes for all violated monitored buses.

Fig. 2 is an example of monitored buses for a DER-connected bus, bus $D G_{2}$. Besides itself, bus $D G_{2}$ has three additional monitored buses, including one upstream monitored bus, $M_{2-U P}$, and two downstream monitored buses $M_{2-D N 1}$, and $M_{2-D N 2}$. The bus $D G_{2}$ adjusts its total reactive power generations from its connected DERs according to the voltages measured on those four monitored buses.

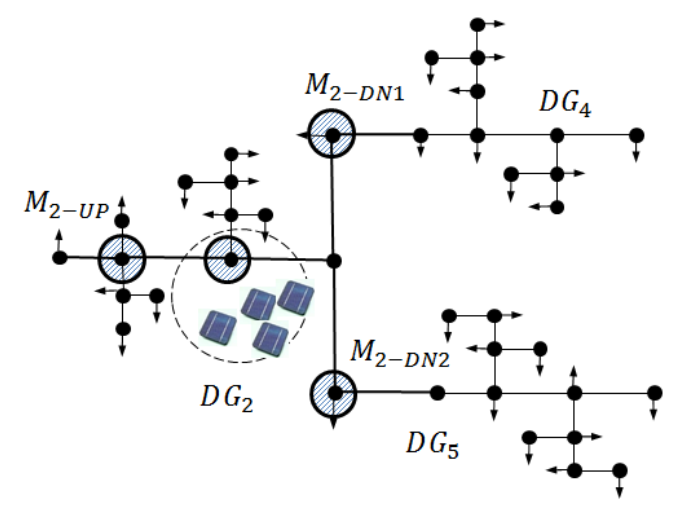

Fig. 2. An example of monitored buses for a DER-connected bus 
For each monitored bus, a set of voltage changes can be determined for clearing its voltage violation according to:

$$
\begin{array}{rlrl}
\Delta\left|V_{i, s}\right|=V_{i}^{\text {min }}-\left|V_{i, s}\right| & & \text { if }\left|V_{i, s}\right|<V_{i}^{\text {min }} \\
\Delta\left|V_{i, s}\right|=0 & \text { if } V_{i}^{\text {min }} \leq\left|V_{i, s}\right| \leq V_{i}^{\text {max }} \\
\Delta\left|V_{i, s}\right|=V_{i}^{\text {max }}-\left|V_{i, s}\right| & \text { if }\left|V_{i, a}\right|>V_{i}^{\text {max }} \\
& \quad s \in\{a, b, c\}
\end{array}
$$

where, $\left|V_{i, s}\right|$ is the magnitude of voltage at bus $i$ on phase $s$, $V_{i}^{\min }$ and $V_{i}^{\max }$ are the lower and upper thresholds for the magnitudes of voltages at bus $i, \Delta\left|V_{i, s}\right|$ is the required voltage change for clearing voltage violation at bus $i$ on phase $s$.

Unlike commonly used sensitivity based approaches that determine the required reactive power changes based on system-wide impedance information and all voltage measurements or estimations, the required changes for reactive powers at any bus are determined based on the voltage violations on the bus, and equivalent impedances and phase angle shifting on the shortest path between the primary side of substation transformer and the bus under consideration.

Fig. 3 gives an example of shortest path between the primary side of substation transformer and a bus, $M_{2-D N 1}$ monitored by the DER-connected bus, $D G_{2}$. It includes 9 line segments.

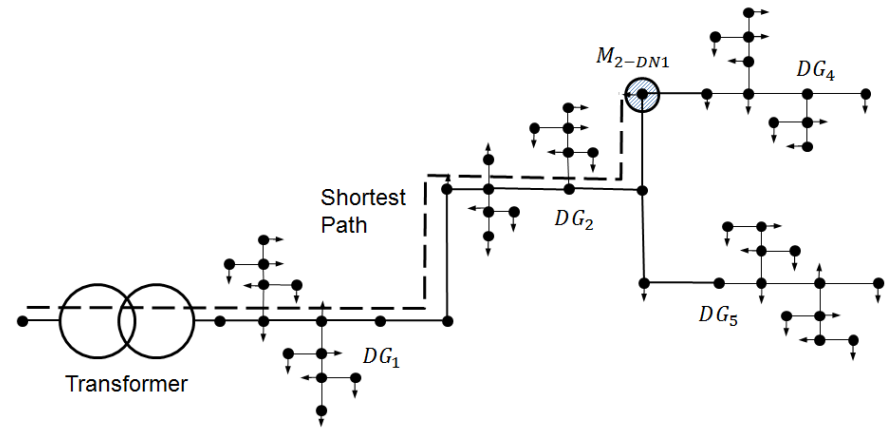

Fig. 3. An example of shortest path between the substation transformer and a monitored bus

The angle shifts between the primary side of substation transformer and bus $i$ on each phase can be determined through an aggregated voltage amplifying factor matrix, $A_{V_{i}}$ that is introduced by the transformers or voltage regulators or phase jumpers along the shortest path between the substation and bus $i$ :

$$
A_{V_{i}}=\prod_{u d} A_{V u d}
$$

where, $A_{V_{u d}}$ is the voltage amplifying factor matrix of a voltage regulator or transformer or phase jumper between two buses, bus $u$ and bus $d$ which reside on the shortest path from the substation to the bus under consideration.

The equivalent impedance of the shortest path between the substation transformer and bus $i, Z_{i}^{E q v}$ are determining by aggregating the impedance matrices for each line segment on the path.

To achieve the voltage changes described in (1), the required current injection changes at bus $i$ are determined as:

$$
\left[\begin{array}{c}
\Delta I_{i, a}^{Q} \\
\Delta I_{i, b}^{Q} \\
\Delta I_{i, c}^{Q}
\end{array}\right]=\left[\begin{array}{ccc}
Z_{i, a a}^{E q v} & Z_{i, a b}^{E q v} & Z_{i, a c}^{E q v} \\
Z_{i, b a}^{E q v} & Z_{i, b b}^{E q v} & Z_{i, b c}^{E q v} \\
Z_{i, c a}^{E q v} & Z_{i, c b}^{E q v} & Z_{i, c c}^{E q v}
\end{array}\right]\left[\begin{array}{c}
\Delta\left|V_{i, a}\right| e^{j\left(\angle A_{V_{i, a}}+0^{o}\right)} \\
\Delta\left|V_{i, b}\right| e^{j\left(\angle A_{V_{i, b}}-120^{\circ}\right)} \\
\Delta\left|V_{i, c}\right| e^{j\left(\angle A_{V_{i, c}}+120^{\circ}\right)}
\end{array}\right]
$$
where, $\Delta\left|V_{i, a}\right|, \Delta\left|V_{i, b}\right|$ and $\Delta\left|V_{i, c}\right|$ are the amount of required voltage changes at bus $i$ on phase $a, b$ and $c ; \angle A_{V_{i, a}}, \angle A_{V_{i, b}}$ and $\angle A_{V_{i, c}}$ are the phase angle shifts between the primary side of substation and bus $i$ on phase $a, b$ and $c$, and given by Eq. (2); $\Delta I_{i, a}^{Q}, \Delta I_{i, b}^{Q}$ and $\Delta I_{i, c}^{Q}$ are the required current injections for phase $a, b$ and $c ; Z_{i}^{E q v}$ is a 3-by-3 matrix of equivalent impedance between the equivalent source at the substation and the monitored bus with voltage violation, and $Z_{i, s t}^{E q v}$ is the element of equivalent impedance matrix at the row corresponding to phase $s$ and the column corresponding to phase $t$.

The reactive power changes at the monitored bus $i$ are determined according to:

$$
\begin{aligned}
\Delta \tilde{Q}_{i, a} & =\operatorname{Im}\left\{\left|V_{i, a}\right| e^{j\left(\angle A_{V_{i, a}}+0^{o}\right)} \Delta I_{i, a}^{Q *}\right\} \\
\Delta \widetilde{Q}_{i, b} & =\operatorname{Im}\left\{\left|V_{i, b}\right| e^{j\left(\angle A_{V_{i, b}}-120^{\circ}\right)} \Delta I_{i, b}^{Q *}\right\} \\
\Delta \tilde{Q}_{i, c} & =\operatorname{Im}\left\{\left|V_{i, c}\right| e^{j\left(\angle A_{V_{i, c}}+120^{o}\right)} \Delta I_{i, c}^{Q *}\right\}
\end{aligned}
$$

where, $\left|V_{i, a}\right|,\left|V_{i, b}\right|$ and $\left|V_{i, c}\right|$ are the measured voltage magnitudes of bus $i$ on phase $a, b$ and $c ; \Delta \tilde{Q}_{i, a}, \Delta \tilde{Q}_{i, b}$ and $\Delta \tilde{Q}_{i, c}$ are the required changes for reactive power injections at bus $i$ on phase $a, b$ and $c$ respectively.

The reactive power changes at DER-connected bus $i$ are determined according to:

$$
\Delta Q_{i, s}=\frac{A_{u} \Delta \tilde{Q}_{u, s}+A_{i} \Delta \tilde{Q}_{i, s}+\sum_{d \in D N_{i}} A_{d} \Delta \tilde{Q}_{d, s}}{A_{u}+A_{i}+\sum_{d \in D N_{i}} A_{d}} \quad s \in\{a, b, c\}
$$

where, $\Delta Q_{i, s}$ is the averaged change of reactive power injection at bus $i$ on phase $s ; A_{u}, A_{i}$ and $A_{d}$ are the availability of voltage violation at bus $u$, bus $i$ and $d$; bus $u$ and bus $d$ are the monitored buses located upstream and downstream to bus $i$ respectively; $D N_{i}$ is the set of monitored buses directly downstream to bus $i$. $\Delta \tilde{Q}_{u, s}$ and $\Delta \tilde{Q}_{d, s}$ are the required changes of reactive power injection at bus $u$ and $d$ on phase $s$.

After obtained the required reactive power changes, the required total reactive powers from bus $i$ on phase $s, \pi_{i s}$ can be determined as:

$$
\pi_{i s}=Q_{i s}^{0}+\alpha \Delta Q_{i, s}
$$

where, $Q_{i s}^{0}$ is the current reactive power at bus $i$ on phase $s$, and $\alpha$ is a scale factor used for preventing reactive power over-compensation when there are multiple violations existing, and can be set as the inverse of total number of DER-buses downstream to the substation.

The reactive power generation at bus $i$ on phase $s, Q_{i, s}$ is bounded by its upper and lower thresholds as:

$$
Q_{i s}^{\min } \leq Q_{i, s} \leq Q_{i s}^{\max }
$$

where, $Q_{i s}^{\max }$ and $Q_{i s}^{\min }$ are the upper and lower thresholds for reactive powers at bus $i$ on phase $s$. There are two factors that constrain the capacity of a DER-connected bus for reactive 
power generation, one is that the total apparent power cannot exceed the rated capacity, and the other is that the power factor must be greater than a minimum allowed value. Accordingly, the upper and lower thresholds for reactive powers at bus $i$ on phase $s$ can be defined as:

$$
\begin{gathered}
Q_{i s}^{\max }=\min \left\{\sqrt{\left(S_{i, s}^{\max }\right)^{2}-\left(P_{i, s}\right)^{2}},\left|P_{i, s}\right| \frac{\sqrt{1-\left(p f^{\min }\right)^{2}}}{p f^{\min }}\right\} \\
Q_{i s}^{\min }=-Q_{i s}^{\max }
\end{gathered}
$$

where, $P_{i, s}$ is the current active power generation at bus $i$ on phase $s, S_{i, s}^{\max }$ is the maximum apprarent power generation at bus $i$ on phase $s$, and $p f^{\min }$ is the minimum allowed power factor.

The DERs directly connected to the bus will be instructed to provide the estimated reactive powers defined in (8) if the estimations are within thresholds given in (10) and (11). Otherwise, they will output their maximum/minimum capacity. The difference in reactive power between the estimate and what the DERs connected to the bus can provide will be requested from other buses that have additional capacities.

\section{Distributed Control of DER-Bus Controllers AND ITERATIVE RESIDUAL SPLITTING ALgORITHM}

The controlling of voltages of the distribution system can be implemented using a two-level control architecture. The first one is the DER-bus controller that determines reactive power needs for each DER-connected bus based on voltage violation in the system. The second one is the DER-unit controller that allocates the reactive power request from a DER-bus into each DER-unit connected to the bus. Each phase of a DER-bus or DER-unit is regulated independently.

\section{A. Distributed Control for DER-bus Controllers}

Each bus connected with DERs is equipped with a local bus controller that regulates the reactive power generations of DERs connected to the bus based on the local measured voltages on its monitored buses, and communicates with neighbor bus controllers located at its upstream and downstream to obtain system-wide information and coordinate the reactive powers between all DER-connected buses.

Fig. 4 gives the schematic of communication paths between DER-connected buses for the distribution system in Fig.1. In Fig. 4, each bus controller has a bi-way communication with its neighbor bus controllers located at its upstream and downstream. Taken the controller at bus $D G_{2}$ as an example, it has bi-way communications with the controller of bus $D G_{1}$ at its upstream, and the controllers of bus $D G_{4}$ and bus $D G_{5}$ at its downstream. For the controller at bus $D G_{1}$, it does not have bus controller at its upstream, so it only communicates with the controllers at its downstream. For the controllers at bus $D G_{4}$, bus $D G_{5}$, and bus $D G_{6}$, they do not have bus controllers at their downstream, so those controllers only communicate with the ones at their upstream.

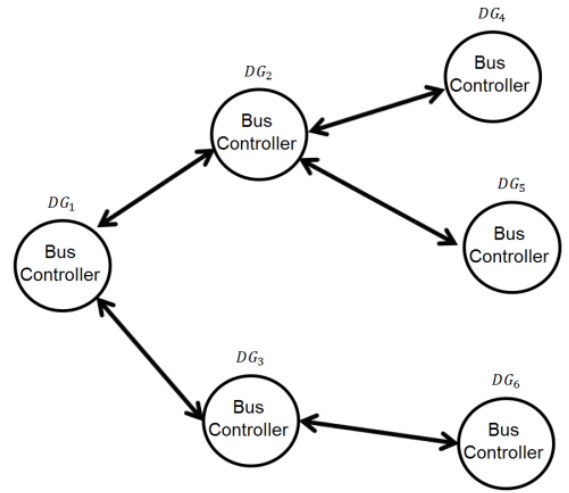

Fig. 4. An example of communication paths between DER-connected buses

The reactive power generation for any DER-connected bus $i$ on any phase $s, Q_{i, s}$ can be divided into three components:

$$
Q_{i, s}=Q_{i, s}^{l o c}+Q_{i, s}^{c a p}-Q_{i, s}^{i n d} \quad s \in\{a, b, c\}
$$

where, $Q_{i, s}^{\text {loc }}$ is the reactive power used to support its own voltage, $Q_{i, s}^{c a p}$ and $Q_{i, s}^{i n d}$ are the additional capacitive and inductive reactive powers used to assist mitigating the over upper- and lower- voltage thresholds of neighbor bus controllers.

The reactive power for supporting its own voltage is determined solely by the local controller at bus $i$ according to:

$$
\begin{array}{ll}
Q_{i, s}^{\text {loc }}=Q_{i, s}^{\min } & \text { if } \pi_{i s}<Q_{i, s}^{\min } \\
Q_{i, s}^{\text {loc }}=\pi_{i s} & \text { if } Q_{i, s}^{\min } \leq \pi_{i s} \leq Q_{i, s}^{\max } \\
Q_{i, s}^{\text {loc }}=Q_{i, s}^{\max } & \text { if } \pi_{i s}>Q_{i, s}^{\max } \\
\quad & \quad s \in\{a, b, c\}
\end{array}
$$

However, the additional capacitive and inductive reactive powers have to be determined through iterative processes for coordination among DER-connected buses in the system using distributed control algorithm.

The initial setting of capacitive reactive power for mitigating lower-threshold voltage violations at its neighbor buses is determined according to:

$$
\begin{array}{lr}
Q_{i, s}^{c a p}[0]=0 & \text { if } \pi_{i s} \leq Q_{i, s}^{\max } \\
Q_{i, s}^{c a p}[0]=\pi_{i s}-Q_{i, s}^{\max } & \text { if } \pi_{i s}>Q_{i, s}^{\max } \\
& s \in\{a, b, c\}
\end{array}
$$

The capacities of the capacitive reactive powers are determined as:

$$
\begin{array}{lr}
Q_{i, s}^{\text {cap, max }}=Q_{i, s}^{\max }-\pi_{i s} & \text { if } \pi_{i s}<Q_{i, s}^{\max } \\
Q_{i, s}^{\text {cap, } \text { max }}=0 & \text { if } \pi_{i s} \geq Q_{i, s}^{\max } \\
& s \in\{a, b, c\}
\end{array}
$$

The initial setting of inductive reactive power for mitigating upper-threshold voltage violations at its neighbor buses is determined according to:

$$
\begin{array}{lr}
Q_{i, s}^{\text {ind }}[0]=Q_{i, s}^{\text {min }}-\pi_{i s} & \text { if } \pi_{i s}<Q_{i, s}^{\text {min }} \\
Q_{i, s}^{\text {ind }}[0]=0 & \text { if } \pi_{i s} \geq Q_{i, s}^{\text {min }} \\
& s \in\{a, b, c\}
\end{array}
$$

The capacities of inductive reactive powers are determined as: 


$$
\begin{array}{lc}
Q_{i, s}^{\text {ind,max }}=0 & \text { if } \pi_{i s}<Q_{i, s}^{\text {min }} \\
Q_{i, s}^{\text {ind,max }}=\pi_{i s}-Q_{i, s}^{\text {min }} & \text { if } \pi_{i s} \geq Q_{i, s}^{\text {min }} \\
& s \in\{a, b, c\}
\end{array}
$$

\section{B. Proposed Iterative Residual Splitting algorithm}

For each phase of each DER-connected bus, its additional capacitive and inductive reactive power generations are separately determined by using a distributed control algorithm described here.

Unlike the common used consensus based algorithms that allocate the total demand among participating parties evenly or proportional to their capacities, this algorithm allocates the total demand more favorable to the buses that are close to the buses with the demand. For a distribution system, the voltage violations are more effectively corrected by the reactive power sources close to the buses with violations. The farther the source from the buses with violations, the less effective the source corrects the voltage violations. Thus, the available even splitting strategy based consensus algorithms, in our opinion, did not well fit with reactive power control problem.

Let $x_{i}$ be the positive reactive power determined for bus $i$, and the controller at the bus is treated as one node of a directed graph. The goal of this algorithm is to find a feasible solution that enables its value within a set of lower and upper bounds:

$$
0 \leq x_{i}^{\min } \leq x_{i} \leq x_{i}^{\max }
$$

where, $x_{i}^{\max }$ and $x_{i}^{\text {min }}$ is the non-negative maximum and minimum capacities of node $i . x_{i}^{\max }$ is defined by (15) for determining the capacitive reactive powers, and (17) for determining the inductive reactive powers. $x_{i}^{\min }$ is set as zero.

The proposed algorithm divides the corresponding reactive power of each bus into two parts, one is a feasible part that lies within its upper and lower bounds, $x_{i}$, and the other is a residual part, $\Delta x_{i}$ that is the amount of reactive powers beyond the bounds. An iterative process is used to update the feasible and residual powers of each node, and split the sum of residual powers between neighbor nodes iteratively until a converged solution is obtained. For practical consideration, the process is terminated when a given number of iterations is reached.

At each step of iterations, the current residual value of each node is split into half for the node itself, and half for the nodes that can receive information from the study node. For example, the current feasible and residual values of node $i$ at current step $k$ are $x_{i}[\mathrm{k}]$ and $\Delta x_{i}[\mathrm{k}]$. The number of neighbor nodes that node $i$ can transmit information to is $D_{i}^{\text {out }}$. The shares for node $i$ and any node adjacent to node $i$ of $\Delta x_{i}[\mathrm{k}]$ are $\frac{1}{2} \Delta x_{i}[\mathrm{k}]$ and $\frac{1}{2 D_{i}^{\text {out }}} \Delta x_{i}[\mathrm{k}]$. The new feasible and residual values for node $i$ at next step $(k+1)$ can be determined by checking against its lower and upper bounds with an aggregate value determined by aggregating its current feasible value and all residual shares allocated from itself, and all nodes that can send information to node $i$ from current step $k$ together. This aggregate value is determined as $\left(x_{i}[\mathrm{k}]+\frac{1}{2} \Delta x_{i}[\mathrm{k}]+\sum_{j \in N_{i}^{\text {in }}} \frac{1}{1+D_{j}^{\text {out }}} \Delta x_{j}[\mathrm{k}]\right), N_{i}^{\text {in }}$ is the set of nodes that can transmit information to node $i$, $\Delta x_{j}[\mathrm{k}]$ is the residual value of node $j$ at step $k, D_{j}^{\text {out }}$ is the number of nodes that node $j$ can transmit information to. The portion within the bounds is the new feasible value, and the portion beyond the bounds is the new residual value.

The feasible and residual powers are iteratively updated according to:

$$
\begin{array}{rlrl}
\widetilde{x}_{l}[\mathrm{k}+1] & \left.=x_{i}[\mathrm{k}]+\frac{1}{2} \Delta x_{i}[\mathrm{k}]+\sum_{j \in N_{i}^{\text {in }}} \frac{1}{1+D_{j}^{\text {out }}} \Delta x_{j}[\mathrm{k}]\right) \\
x_{i}[\mathrm{k}+1] & =x_{i}^{\text {min }} & & \text { if } \widetilde{x_{l}}[\mathrm{k}+1]<x_{i}^{\text {min }} \\
x_{i}[\mathrm{k}+1] & =\widetilde{x}_{l}[\mathrm{k}+1] & & \text { if } x_{i}^{\text {min }} \leq \widetilde{x_{l}}[\mathrm{k}+1] \leq x_{i}^{\text {max }} \\
x_{i}[\mathrm{k}+1] & =x_{i}^{\text {max }} & & \text { if } \tilde{\tilde{x}_{l}}[\mathrm{k}+1]>x_{i}^{\text {max }} \\
\Delta x_{i}[\mathrm{k}+1] & =\widetilde{x_{l}}[\mathrm{k}+1]-x_{i}^{\text {min }} \quad & \text { if } \tilde{x_{l}}[\mathrm{k}+1]<x_{i}^{\text {min }} \\
\Delta x_{i}[\mathrm{k}+1] & =0 & & \text { if } x_{i}^{\text {min }} \leq \widetilde{x_{l}}[\mathrm{k}+1] \leq x_{i}^{\text {max }} \\
\Delta x_{i}[\mathrm{k}+1] & =\widetilde{x_{l}}[\mathrm{k}+1]-x_{i}^{\text {max }} \quad \text { if } \widetilde{x_{l}}[\mathrm{k}+1]>x_{i}^{\text {max }}
\end{array}
$$

where, $\widetilde{x_{l}}[\mathrm{k}+1]$ is an aggregation of current feasible power and shares of residual powers. $x_{i}[\mathrm{k}+1]$ and $\Delta x_{i}[\mathrm{k}+1]$ are the feasible and residual reactive powers of bus $i$ at step $(k+1)$. The initial feasible and residual powers at bus $i, x_{i}[0]$, and $\Delta x_{i}[0]$ are determined based on the reactive powers defined by (14) for determining the capacitive reactive powers, and (16) for determining the inductive reactive powers.

After a given number of iterations, the additional capacitive, or inductive reactive power supplied by bus $i, x_{i}$ can be set as:

$$
x_{i}=x_{i}[m]
$$

where, $m$ is a predetermined number.

Using this algorithm, each DER-bus controller only needs local information from itself and neighbors to make decisions. At each iterative step, each bus only needs to send the amount of shares for its residual reactive power to its neighbor buses, and receives the shares of other bus's residual active powers from its neighbor buses.

\section{Distributed CONTROL OF DER-Unit CONTROLLERS USING CONSENSUS BASED ALGORITHM}

After the DER-bus controller determined the amount of reactive powers that the DERs connected to the bus need to be generated, the DER-bus controller will send the amount of required reactive powers to the DER units, and the request will be allocated to each unit according to their capacities using a consensus based distributed control algorithm.

\section{A. Distributed Control for DER-unit Controllers}

Each individual unit at the bus connected with DERs has an independent unit controller for adjusting its reactive power injections according to the total amount of reactive power 
determined for the bus by the bus controller and generations from all other units connected to the same bus.

Fig. 5 gives an example of communication paths between a DER-bus controller and corresponding unit controllers for the DER units connected to the bus. The bus, $D G_{1}$ has connected with four units, $D G_{1-1}, D G_{1-2}, D G_{1-3}$ and $D G_{1-4}$. Each unit has two-way communications with its neighbor units. One of the units is selected as the leading unit to receive command from the bus controller. For example, unit $D G_{1-1}$ is selected as the leading unit in Fig. 5.

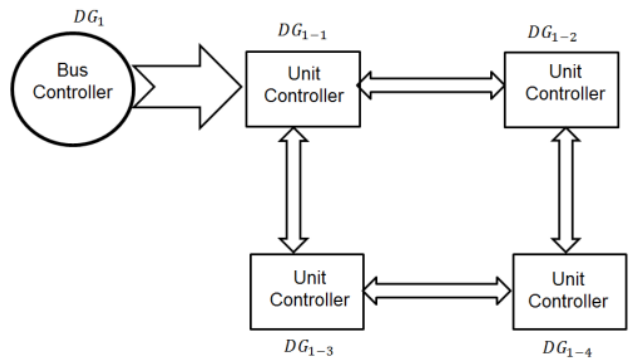

Fig. 5. An example of communication paths between DER-unit controllers and local bus controller

The bus controller issues a command demanding an amount of reactive power to a leading unit controller. After the leading unit receives the command, an iterative exchange of information between units begins: each unit in the network exchanges information with its neighboring units. The objective of this information exchange is to distribute the required amount of reactive power among all the units such that after several rounds of exchanges, each unit keeps a fraction of total demand, such that collectively the units provide the total amount of reactive power demanded by the bus controller.

\section{B. Fine-time Consensus-based Algorithm}

A consensus based distributed control algorithm [8] is used here. Each unit controller is treated as a node of a directed graph. The algorithm has executed two iterative processes in parallel. The goal of the first process is evenly splitting the total demand into each node, and the second one is evenly splitting the sum of unit capacities into each node.

Let $y_{i}$ be the positive reactive power of node $i$, and its feasible value is constrained by an non-negative lower bound, $y_{i}^{\min }$ and an non-negative maximum bound, $y_{i}^{\max }$. The initial condition for the reactive power of node $i$ is given by $y_{i}[0]$. The first iterative process is used to update the reactive powers of units according to:

$$
y_{i}^{1}[k+1]=\sum_{j \in\left\{i \cup N_{i}^{i n}\right\}} \frac{1}{1+D_{j}^{\text {out }}} y_{i}^{1}[k]
$$

where, $y_{i}^{1}[k]$ and $y_{i}^{1}[k+1]$ are the values of reactive powers of node $i$ at the step $k$, and step $(k+1)$. The initial condition of $y_{i}^{1}[k]$ is set as:

$$
y_{i}^{1}[0]=y_{i}[0]-y_{i}^{\min }
$$

The second iterative process is used to update the capacities of nodes according to:

$$
y_{i}^{2}[k+1]=\sum_{j \in\left\{i \cup N_{i}^{i n}\right\}} \frac{1}{1+D_{j}^{\text {out }}} y_{j}^{2}[k]
$$

where, $y_{i}^{2}[k]$ and $y_{i}^{2}[k+1]$ are the values of capacities of node $i$ at the step $k$, and step $(k+1)$. The initial condition of $y_{i}^{2}[k]$ is set as:

$$
y_{i}^{2}[0]=y_{i}^{\max }-y_{i}^{\min }
$$

After specified number of iterations, $m$, the final solution for reactive power supplied by node $i, y_{i}$ can be determined as:

$$
y_{i}=y_{i}^{\min }+\frac{y_{i}^{1}[\mathrm{~m}]}{y_{i}^{2}[\mathrm{~m}]}\left(y_{i}^{\max }-y_{i}^{\min }\right)
$$

Depending on the configuration of communication between unit controllers, the algorithm does not necessarily split the demand among all units proportional to unit capacities but it does ensure that the overall demand is collectively met and the reactive powers being demanded from each unit does not exceed its capacity limit if the total demand is not greater than the total capacity limit. If there is no enough information for determining whether the total demand is less than total capacity limit, the reactive power supplied by each unit will be checked against its capacity limit, and set to its value as its limit when it is over the limit.

\section{NUMERICAL EXAMPLES}

The proposed distributed control method has been tested on a 123-bus system with a significant penetration of PV installations.

The test system was adapted from the IEEE 123-node feeder [9], and its one-line diagram is given in Fig. 6. The system is modified to include 6 buses connected with DERs, and each bus has installed 4 photovoltaic units. The upper and lower thresholds for phase voltages are set as 1.05 and 0.95 per unit respectively.

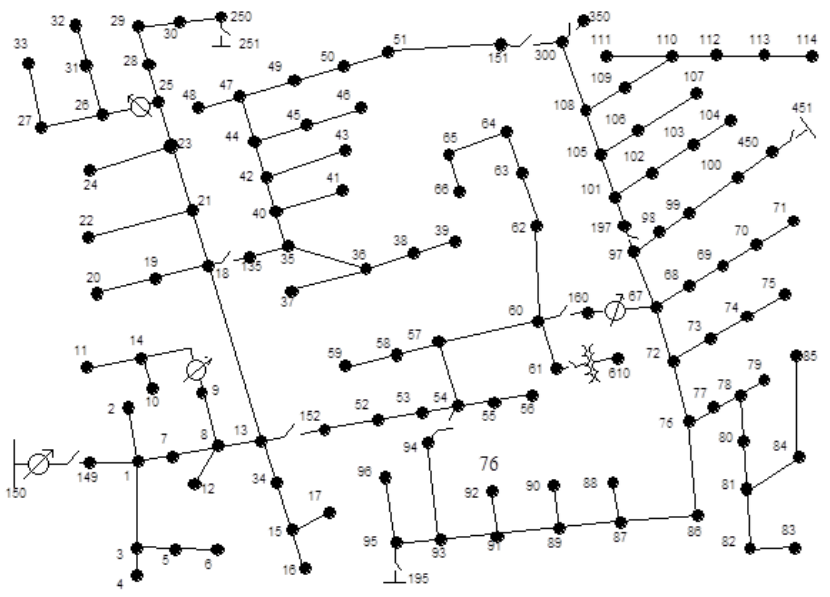

Fig. 6. One line diagram for IEEE 123-node feeder

Table I lists the detailed information for DER-buses in the system, including their monitored buses, total number of connected photovoltaic units, scheduled active power 
generations for each unit on each phase, maximum capacities and allowed minimum power factors. Table II gives the communication paths between DER-bus controllers in the system. Table III gives the communication paths between DER units of each DER-bus.

TABLE I

BUSES CONNECTED WITH DERS

\begin{tabular}{|c|c|c|c|c|c|c|}
\hline Bus & $\begin{array}{c}\text { Monitored } \\
\text { Buses }\end{array}$ & Units & Phases & $\begin{array}{c}\text { Sch. } \\
\text { Gen. } \\
\text { (KVA } \\
\text { /unit } \\
\text { phase) }\end{array}$ & $\begin{array}{c}\text { Cap. } \\
\text { (KVA } \\
\text { /Unit } \\
/ \text { Phase) }\end{array}$ & $\begin{array}{c}\text { Min. } \\
\text { Pow. } \\
\text { Factor }\end{array}$ \\
\hline 13 & $1,16,18,53$ & 4 & $\mathrm{ABC}$ & $40+\mathrm{j} 0$ & 50 & 0.7 \\
\hline 35 & $18,39,42$ & 4 & $\mathrm{ABC}$ & $40+\mathrm{j} 0$ & 50 & 0.7 \\
\hline 47 & $42,48,51$ & 4 & $\mathrm{ABC}$ & $40+\mathrm{j} 0$ & 50 & 0.7 \\
\hline 57 & $53,59,62,67$ & 4 & $\mathrm{ABC}$ & $100+\mathrm{j} 0$ & 150 & 0.7 \\
\hline 64 & 62,66 & 4 & $\mathrm{ABC}$ & $100+\mathrm{j} 0$ & 150 & 0.7 \\
\hline 76 & $67,83,85,93$ & 4 & $\mathrm{ABC}$ & $40+\mathrm{j} 0$ & 50 & 0.7 \\
\hline 101 & $67,104,107,114$ & 4 & $\mathrm{ABC}$ & $40+\mathrm{j} 0$ & 50 & 0.7 \\
\hline
\end{tabular}

TABLE II

Bus CONTROLLER COMMUNICATION PATHS

\begin{tabular}{|cc|cc|}
\hline Sending Bus & Receiving Bus & Sending Bus & Receiving Bus \\
\hline 13 & 35 & 57 & 64 \\
\hline 13 & 57 & 57 & 76 \\
\hline 35 & 13 & 57 & 101 \\
\hline 35 & 47 & 64 & 57 \\
\hline 47 & 35 & 76 & 57 \\
\hline 57 & 13 & 101 & 57 \\
\hline
\end{tabular}

TABLE III

UNIT CONTROLLER COMMUNICATION PATHS

\begin{tabular}{|cc|cc|}
\hline Sending Unit & Receiving Unit & Sending Unit & Receiving Unit \\
\hline 1 & 2 & 2 & 4 \\
\hline 1 & 3 & 3 & 1 \\
\hline 2 & 1 & 4 & 2 \\
\hline
\end{tabular}

Fig. 7 gives a daily load profile and a daily generation profile that used for all loads and distributed resources in the system. The sampling rate is once per 3 minutes. The horizontal axis and vertical axis represent the accumulated number of intervals, and scaling factors for loads and active-power generations respectively. The base loads are given in [9], and base generations are given in Table I.

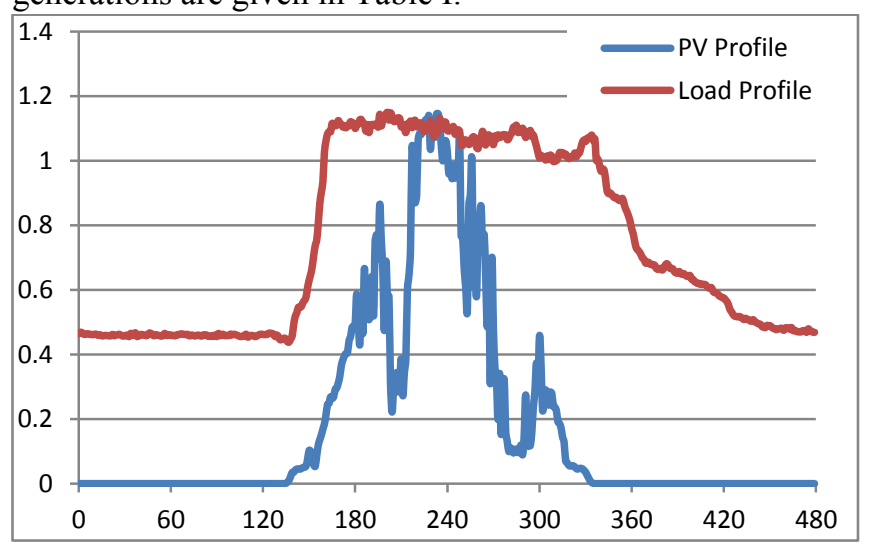

Fig. 7. Daily load profile and generation profile
Both the centralized method and the proposed distributed method are implemented for the test system. The centralized method determines the reactive powers based on the Jacobian matrices, and utilizes the system wide voltage measurements. It has been verified that the proposed method can produce the similar results as the centralized one.

Fig. 8 gives the 24-hour curves of system highest and lowest voltages on each phase when the distributed resources operated at the unity power factor mode. In this mode, the distributed resources only provide active power generations for the system. As shown in Fig. 8, the voltage violations for the upper bounds are occurring during several periods that the PV units generate active powers.

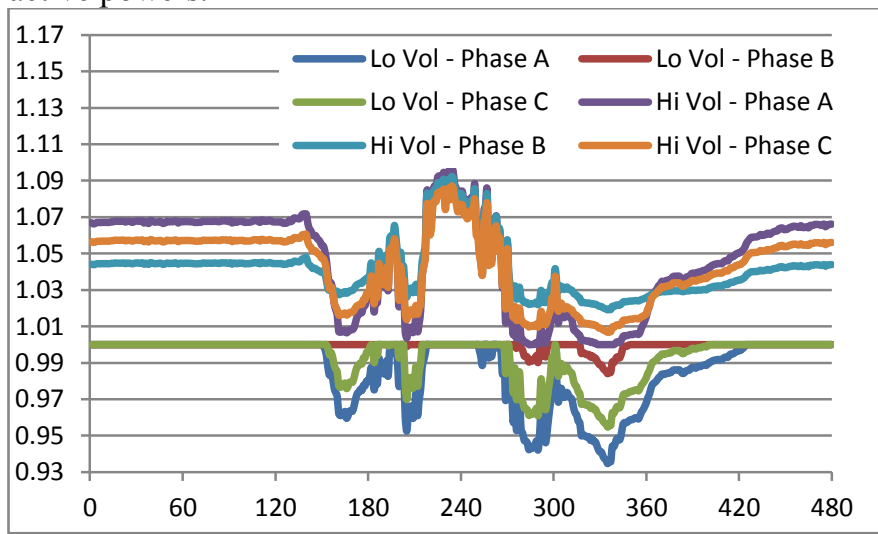

Fig. 8. System highest and lowest voltages when DERs operated with unity power factors

Fig. 9 gives the 24-hour curves for system highest and lowest voltages on each phase when the distributed resources are operating at the reactive power resource mode. In this mode, the distributed resources provide both active power and reactive power generations to the system. It is obvious that the voltage violations caused by the PV units as shown in Fig. 8 have been significantly mitigated through regulating the reactive powers of distributed resources.

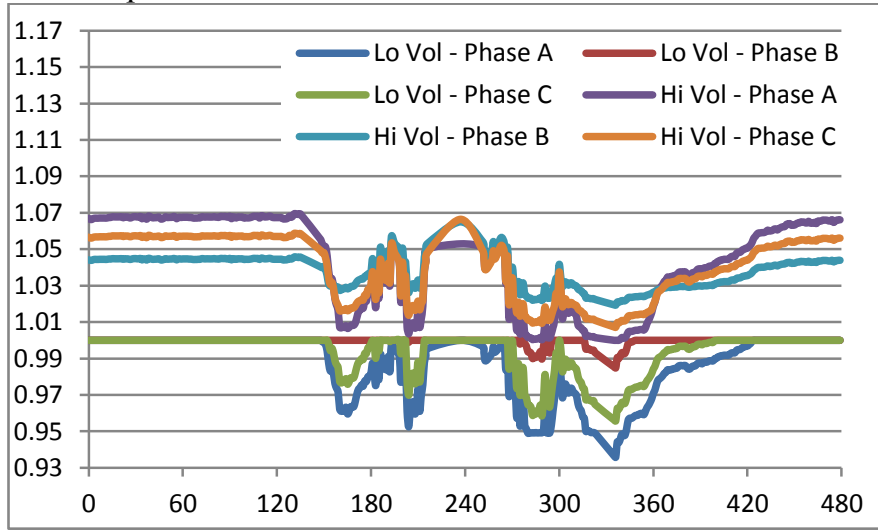

Fig. 9. System highest and lowest voltages when DERs operated with adjustable reactive powers

Fig. 10 gives the corresponding 24-hour curves for total reactive powers generated by all distributed resources in the 
system when operated at the reactive power resource mode.

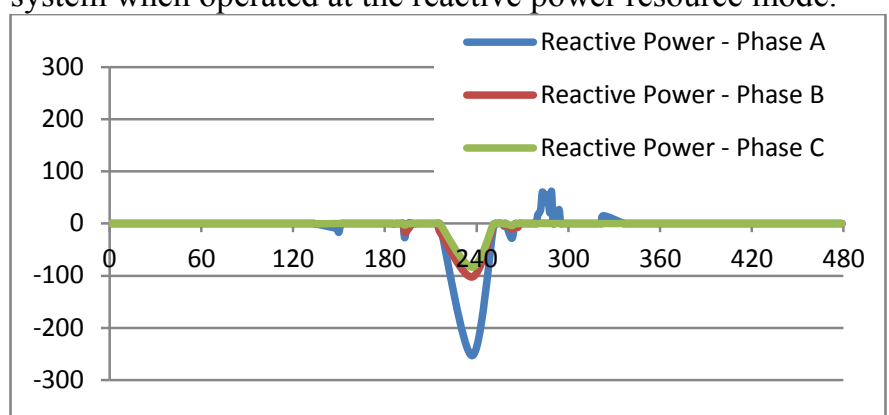

Fig. 10. Total reactive powers generated by DERs when DERs operated with adjustable reactive powers

Based on those preliminary results, it is safe to say that the proposed distributed control method has effectively regulated the reactive powers of DERs to control the system voltages that usually require system-wide information for a centralized method.

\section{CONCLUSIONS}

This paper has proposed a distributed control method for controlling voltages of distribution systems through regulating three-phase reactive powers of distributed energy resources. Unlike the Jacobian matrix based method that requires system-wide voltage information, the proposed method determines the reactive power changes for a DER-connected bus based on the measured voltages on its local monitored buses, and equivalent impedances and phase angle shifting between the substation transformer and the bus under consideration.

A bus controller is equipped to each DER-connected bus to adjust the reactive power generations of DERs connected to the bus, and only communicates with its neighboring bus controllers at its upstream and downstream. A DER-connected bus adjusts its reactive powers up to its capacities to prevent voltage violations on its monitored buses, and an iterative coordination between all DER-buses is used when the required reactive powers for any DER-bus are over its capacities. Unlike consensus based method that allocates total demand evenly or proportional to their capacities among participating parties, the proposed residual splitting method allocates total required reactive powers more favorable to the DER-buses that are close to the buses with violations.

A DER-unit controller is equipped to each DER unit at a DER-connected bus, and uses a bi-way communication to exchange information with its adjacent units. The total reactive power request of the bus is allocated to all units according to their capacities.

\section{REFERENCES}

[1] "IEEE application guide for IEEE Std 1547, IEEE standard for interconnecting distributed resources with electric power systems", IEEE Std 1547.2-2008, pp. 1-207, April 2009.
[2] M. E. Baran and I. M. El-Markabi, "A multiagent-based dispatching scheme for distributed generators for voltage support on distribution feeders", IEEE Trans. Power Sys., vol. 22, no. 1, pp. 52-59, Feb. 2007.

[3] K. Rogers, R. Klump, H. Khurana, A. Aquino-Lugo, and T. Overbye, "An authenticated control framework for distributed voltage support on the smart grid", IEEE Trans. Smart Grid, vol. 1, no. 1, pp. 40-47, June 2010.

[4] D. Villacci, G. Bontempi, and A. Vaccaro, "An adaptive local learning based methodology for voltage regulation in distribution networks with dispersed generation", IEEE Trans. Power Sys., vol. 21, no. 3, pp. 11311140, Aug. 2006.

[5] P. Vovos, A. Kiprakis, A. Wallace, and G. Harrison, "Centralized and distributed voltage control: Impact on distributed generation penetration", IEEE Trans. Power Sys., vol. 22, no. 1, pp. 476-483, Feb. 2007.

[6] E. Polymeneas and M. Benosman, "Finite Time Protocols for Multi-Agent Control of Distributed Generation and Responsive Loads", in Proc. of IEEE American Control Conference, pp. 1469-1474, Portland, Oregon, USA, 2014.

[7] B.A. Robbins, C.N. Hadjicostis; and A.D. Dominguez-Garcia, "A Two-Stage Distributed Architecture for Voltage Control in Power Distribution Systems", IEEE Trans. on Power Sys., vol.28, no.2, pp. 1470,1482 , May 2013

[8] S. T. Cady, A. D. Domínguez-García, and C. N. Hadjicostis, "Robust implementation of distributed algorithms for control of distributed energy resources", in Proc. of North American Power Symposium, Boston, MA, August 2011.

[9] IEEE PES. (2010, Sept.) Distribution test feeders. [Online]. Available: http://www.ewh.ieee.org/soc/pes/dsacom/testfeeders/index.html

Hongbo Sun received a Ph.D. degree in Electrical Engineering from Chongqing University, China in 1991. He is a senior member of IEEE. He is currently working at Mitsubishi Electric Research Laboratories in Cambridge, Massachusetts, USA. His research interests include power system planning and analysis, power operation and control, and smart grid applications.

Mouhacine Benosman received a Ph.D. degree in Control Theory from Ecole Centrale de Nantes, France in 2002. He is a senior member of IEEE. He is currently working at Mitsubishi Electric Research Laboratories in Cambridge, Massachusetts, USA. His research interests include non-linear robust and fault tolerant control, and multi-agent control with applications to smart-grid.

Daniel Nikovski received a Ph.D. degree in Robotics from Carnegie Mellon University in Pittsburgh, USA, in 2002. He is currently working at Mitsubishi Electric Research Laboratories in Cambridge, Massachusetts, USA. His research interests include machine learning, optimization and control, and numerical methods for analysis of complex industrial systems.

Jinyun Zhang received her Ph.D. in Electrical Engineering from the University of Ottawa, Canada in 1991. She is a fellow of IEEE. She is currently working at Mitsubishi Electric Research Laboratories in Cambridge, Massachusetts, USA. Her research interests include digital signal processing, wireless communications, optical networks, and smart grid applications.

Tomihiro Takano received M.S degree in precision engineering from Kyoto University in 1989. He is a senior member of IEEJ, a member of SICE, CIGRE. $\mathrm{He}$ is currently working at the Advanced Technology R\&D Center, Mitsubishi Electric Corp. His research interests include operation, control and protection for power systems, distribution resources, micro-grids.

Yasuhiro Kojima received a Ph.D. degree from Osaka University, Japan in 2008. He is a senior member of IEEJ, a member of IEEE. He is currently working at the Advanced Technology R\&D Center, Mitsubishi Electric Corp. His research interests include smart information technology applications for social infrastructure like power systems.

Tetsufumi Ohno received M.S degree in human information engineering from Osaka University in 2009. He is a member of IEEJ. He is currently working at the Advanced Technology R\&D Center, Mitsubishi Electric Corp. His research interests include distribution system analysis and control. 